\title{
Infrared Time Domain Spectroscopy with Synchronized Frequency Combs
}

\author{
Nathan R. Newbury, Ian Coddington, and William C. Swann \\ National Institute of Standards and Technology, 325 Broadway, Boulder, Colorado 80305, USA \\ email: nnewbury@boulder.nis.gov
}

\begin{abstract}
We describe a frequency-comb based system for time-domain spectroscopy in the near infrared. Our configuration implements synchronous, repetitive sampling of the time domain signature for real-time coherent signal averaging and improved signal-to-noise ratio.

This contribution of NIST, an agency of the US government, is not subject to copyright.

OCIS codes: 300.6500 Spectroscopy, time-resolved, 320.7090 Ultrafast lasers
\end{abstract}

\section{Introduction}

The broadband and coherent output of frequency combs can be exploited for spectroscopy by use of two coherent, asynchronous frequency combs.[1-6] There have been several demonstrations of this approach where either both comb sources are transmitted through the sample or only one comb is transmitted. We take the later approach where the second comb source acts solely as a local oscillator (LO) to "read out" the resulting changes in the transmitted comb; in this way both the phase and amplitude of the frequency-domain signal are recorded with respect to the LO. In the time domain, the system provides the full time-dependent signature of the excited sample, i.e. the free induction decay (FID). In this regard, the system is very similar to time domain spectroscopy in the THz or microwave region of the spectrum;[7-11] pulses from the LO source essentially act both to down-mix the signal and as a fast sampling time gate. We will discuss our approach which utilizes a particular phase-locking configuration to enable real-time coherent averaging of the time domain signal for higher signal to noise ratio (SNR). We also show some example free induction decay signals and compare to theory.

\section{Experimental setup and synchronous sampling}

The experimental setup is shown in Figure 1a and follows Refs. [5, 12-14]. The two frequency comb sources produce pulse trains in time. The pulse train from the excitation comb source is split; half of it is transmitted through the cell to provide a "signal" and half of it bypasses the cell to provide a "reference" pulse. The signal and reference pulses are then "read out" by the LO pulse train (see Fig. 1b). Deconvolution of the two pulses isolates the response of the gas molecules.

(a)

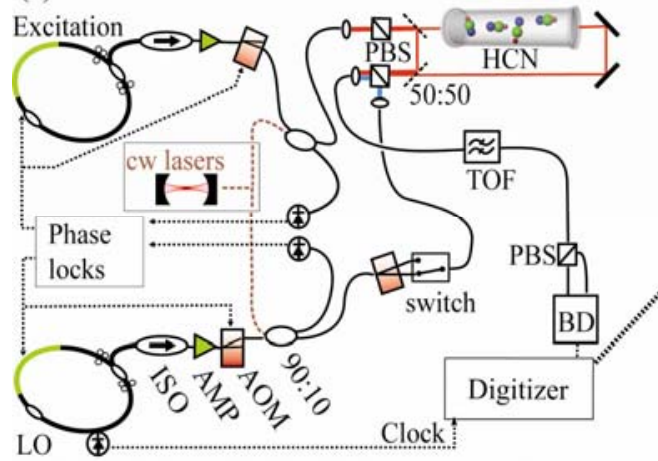

(b)

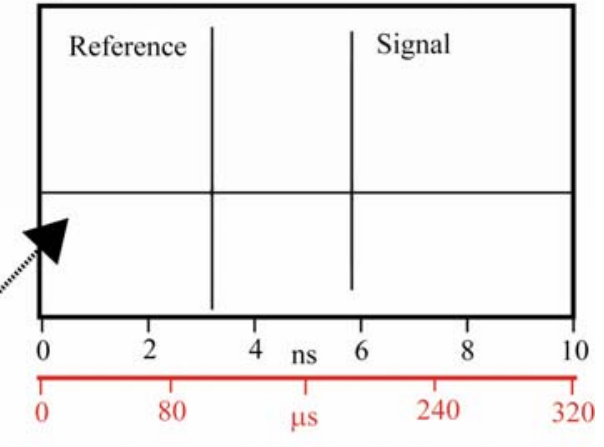

Figure 1. (a) Experimental setup showing the dual fiber-laser based frequency comb sources - an "Excitation" source and a LO source - both are phase-locked to two cw lasers. The sample is a $20 \mathrm{~cm}$ long cell of $2.7 \mathrm{~T}$ hydrogen cyanide (HCN) gas. The excitation pulse train is split to provide a "signal" pulse train that passes through the gas cell and a "reference" pulse train that does not. The LO pulse train acts as a fast, coherent time gate to down sample the reference and signal pulses. (b) Digitized, down-sampled signal showing the reference and signal pulses separated by $2.6 \mathrm{~ns}$ (effective time) in terms of the laboratory time (red, lower axis) and effective time (black, upper axis). As indicated, the LO pulse train completes a single scan of the reference and signal pulses in a time of $\sim 320$ microseconds. To reach reasonable signal-to-noise ratios, many such traces must be coherently added together and the data shown in (b) represents such an average trace. ISO: fiber isolater, AMP: fiber amplifier, AOM: acousto-optic modulator, TOF: tunable optical filter, PBS: polarizing beam splitter, BD: balanced detection. 


\section{LTuA3.pdf}

The basic approach works by locking the two frequency combs to have a slight difference in repetition rate so that the LO pulse train walks through the signal pulse train. For our system, the comb repetition rate is $f_{r}=100 \mathrm{MHz}$ and the difference is $\Delta f_{r}=3 \mathrm{kHz}$. As discussed elsewhere, [1, 3, 4, 6, 13] there is an effective Nyquist limit on the optical bandwidth of the signal that can be measured given values of $f_{r}$ and $\Delta f_{r}$; we set the optical bandpass filter width at $2 \mathrm{~nm}$, which is comfortably below this limit, and acquire data as the bandpass is swept across the spectrum. (A lower $\Delta f_{r}$ would permit a broader bandpass filter but then the peak signal would exceed the dynamic range of the detector.) The data at different filter settings are then coherently stitched together to generate a full bandwidth signal.[14] If the bandpass filter is centered near one of the lock points, or directly between them, then the rf heterodyne signal between the combs occurs at either zero or Nyquist frequency. To avoid these dead zones, we use a fiber switch to redirect the LO pulse train through a modulator in order to shift the LO comb offset frequency by integer multiples of $\Delta f_{r}$. In that way, the rf heterodyne beat is moved away from zero, yet the synchronous sampling, discussed below, is retained.

In order to implement real-time averaging it is critical to control the relative phase-locks between the two comb sources.[13] It is easiest to consider the phase-locking in the frequency domain where the source output forms a frequency comb.[15-17] The sources are stabilized by phase locking a pair of comb teeth (from each comb) to a pair of cw lasers as indicated in Fig 1a. The phase locks are implemented with exactly the same rf offsets; the only difference between the two phase locks is that there are $M$ comb teeth between the two lock points for the LO comb and only $M-1$ comb between the two lock points for the Excitation comb. For these locking conditions, after exactly $(M-1)$ LO pulses, or a time of $\left(\Delta f_{r}\right)^{-1}$ the LO pulse train will have shifted in time relative to the excitation pulse train by exactly one period (of the excitation laser).[13] With a tight phase lock, even the relative optical phase of the two pulses is reproduced. As a result, successive "walk throughs" or "frames" will be identical and can simply be added in time to dramatically increase the SNR without an undue burden on data storage and processing.

Typically, we coherently average, without invoking phase correction, for as long as 3 seconds with negligible signal loss. The SNR is improved by $\sqrt{3 \sec \times \Delta f} \approx 100$ at no cost of additional data storage. Note that without real-time averaging, this same $3 \mathrm{sec}$ acquisition would cost $3 \mathrm{sec} \times 100 \mathrm{Msamples} / \mathrm{sec} \times 2$ bytes $=600 \mathrm{Mbytes}$ of data storage for every $2 \mathrm{~nm}$ of recorded spectra. However, with averaging we get a factor of $10^{4}$ decrease in file size and a corresponding improvement in the FFT-intensive processing time. For longer averaging periods, drifts in the physical path lengths cause carrier shifts and the traces must be phase corrected before adding.

\section{Time domain spectroscopy of free induction decay}

An example of data taken over a $2 \mathrm{THz}$ bandwidth covering P-branch of the HCN first overtone rovibrational lines (near $1550 \mathrm{~nm}$ ) is shown in Figure 2 for a $\sim 2.7$ Torr, $20 \mathrm{~cm}$ long cell. Similar data in the frequency domain, although at lower SNR, is given in Ref. [5]. As shown in Fig. 2 the signal consists of an initial pulse followed by the free induction decay (FID) of the excited molecules. Figures 3 shows a further expanded view of this FID along with a simulation based on HCN molecular parameters. The agreement is excellent between the simulation and the data. The observed structure in the FID occur due to the multiple different rovibrational lines and have been denoted as commensurate echos in THz-TDS or rotational recurrences in rotational pump probe experiments.[8, 18, 19]
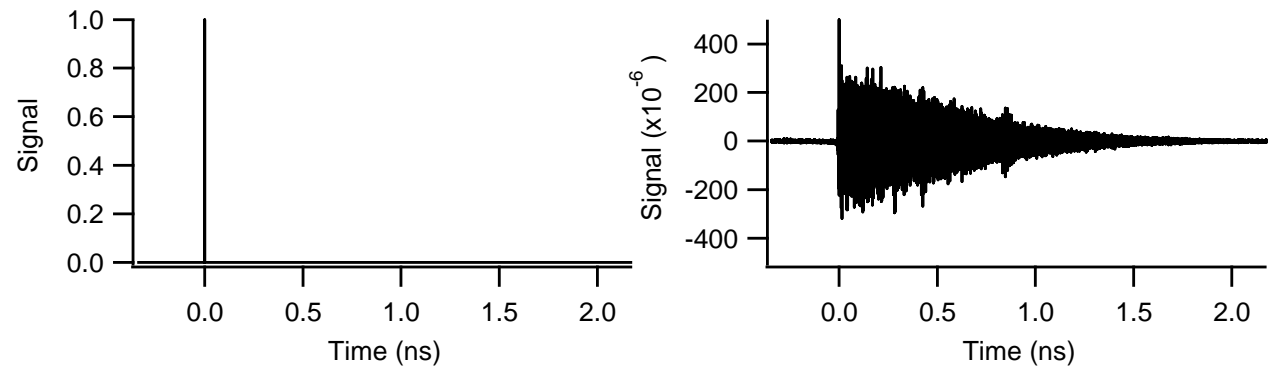

Figure 2: (a) Measured FID decay signal over the P-branch of the HCN rovibrational lines from 192.3 to 194.3 THz. On this scale, only the incident pulse is evident, which is normalized to unity. The FID is too small to be resolved. b) An expanded view of the signal. The main peak is now off scale but the FID is evident, extending out to about 2 ns. The complex structure is due to the different rovibratinal lines. 


\section{LTuA3.pdf}

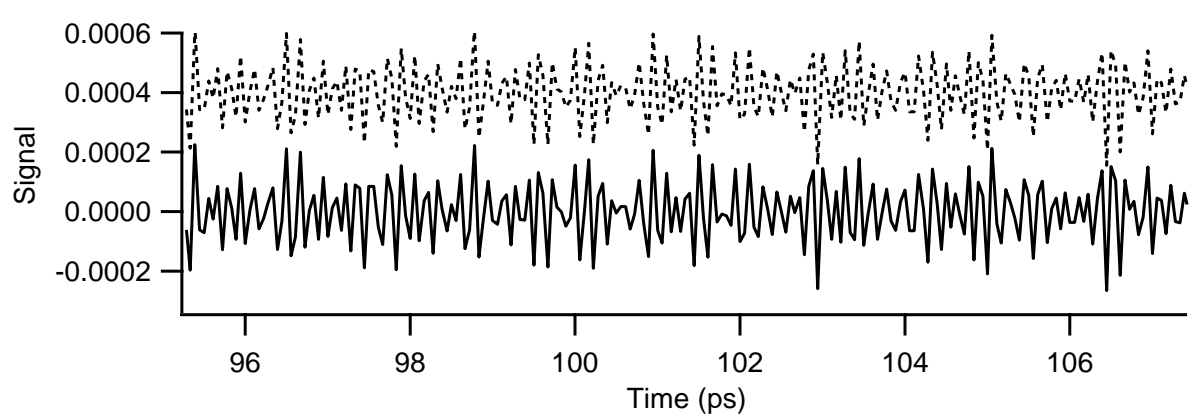

Figure 3: Expanded view of the FID over the time window indicated. The solid line is the data and the dashed line is a simulation based on measured line strengths, collisional linewidths, and calculated Doppler linewidths. The simulation agrees well with the data. Note that the structure on the data is primarily a result of the different contributions of the rovibrational lines and is not noise.

\section{Conclusion}

This system demonstrates the ability to generate the same type of time domain spectroscopic signature in the infrared (or other regions of the optical spectrum) as is possible in the $\mathrm{THz}$ domain. The LO pulse train acts as a coherent short time gate and effectively both down-mixes and time gates the electric field of the signal pulse train to provide a time-domain measurement of the electric field. Through the use of the swept tunable filter and coherent processing, the time gate can have a duration limited only by the bandwidth of the sources. Clearly, to be of practical use, the SNR of the system must be improved to match the impressive levels possible with other spectroscopic techniques. We demonstrate one technique - real time coherent averaging - that leads to improved SNR. Further improvements to the SNR should be possible with higher dynamic range detection, multiplexed detection, mid or far-infrared sources and higher laser powers.

\section{References}

1. F. Keilmann, C. Gohle, R. Holzwarth, Opt. Lett., 29, 1542-1544 (2004).

2. S. Schiller, Opt. Lett., 27, 766-768 (2002).

3. A. Schliesser, M. Brehm, F. Keilmann, D. v. d. Weide, Opt. Express, 13, 9029-9038 (2005).

4. T. Yasui, Y. Kabetani, E. Saneyoshi, S. Yokoyama, T. Araki, Appl. Phys. Lett., 88, 241104 (2006).

5. I. Coddington, W. C. Swann, N. R. Newbury, Phys. Rev. Lett., 100, 013902 (2008).

6. P. Giaccari, J.-D. Deschenes, P. Saucier, J. Genest, P. Tremblay, Opt. Express, 16, 4347-4365 (2008).

7. A. Abragam: The Principles of nuclear magnetism. (Clarendon Press, Oxford, 1961)

8. H. Harde, S. Keiding, D. Grischkowsky, Phys. Rev. Lett., 66, 1834-1837 (1991).

9. D. M. Mittleman, R. H. Jacobsen, R. Neelamani, R. G. Baraniuk, M. C. Nuss, Appl. Phys. B-Lasers Opt., 67, 379-390 (1998).

10. A. Bartels, R. Cerna, C. Kistner, A. Thoma, F. Hudert, C. Janke, T. Dekorsy, Rev. Sci. Instruments, 78, 035107-1 - 035107-8 (2007).

11. B. C. Dian, G. G. Brown, K. O. Douglass, B. H. Pate, Science, 320, 924-928 (2008).

12. I. Coddington, W. C. Swann, L. Nenadovic, N. R. Newbury, Nature Photon., 3, 351-356 (2009).

13. I. Coddington, W. C. Swann, N. R. Newbury, Opt. Lett., 34, 2153-2155 (2009).

14. I. Coddington, W. C. Swann, N. R. Newbury, submitted for publication, (2009).

15. T. Udem, R. Holzwarth, T. W. Hänsch, Nature, 416, 233-237 (2002).

16. D. J. Jones, S. A. Diddams, J. K. Ranka, A. Stentz, R. S. Windeler, J. L. Hall, S. T. Cundiff, Science, 288, 635-9 (2000).

17. N. R. Newbury, W. C. Swann, J. Opt. Soc. Am. B, 24, 1756-1770 (2007).

18. P. M. Felker, J. Phys. Chem., 96, 7844-7857 (1992).

19. J. S. Baskin, A. H. Zewail, J. Phys. Chem. A, 105, 3680-3692 (2001). 